'Departamento de Medicina Interna, Facultad de Medicina, Pontificia Universidad Católica de Chile. Santiago, Chile.

${ }^{2}$ Departamento de Medicina Interna, Programa de Geriatría, Facultad de Medicina, Pontificia Universidad Católica de Chile. Santiago, Chile.

${ }^{3}$ Departamento de Medicina Interna, Grupo de Medicina Interna Hospitalaria, Facultad de Medicina, Pontificia Universidad Católica de Chile. Santiago, Chile.

aAlumno de la carrera de Medicina, Facultad de Medicina, Pontificia Universidad Católica de Chile. Santiago, Chile.

Los autores declaran no tener conflictos de interés. Trabajo no recibió financiamiento.

Recibido el 8 de agosto de 2017, aceptado el 19 de junio de 2018.

Correspondencia a: Dr. Gonzalo Eymin Lago Hospital Clínico Red de Salud UC- Christus Marcoleta $367,5^{\circ}$ piso, Santiago, Chile Fono: +56223543152 geymin@med.puc.cl

\section{Prevalencia de pacientes con alto riesgo de caídas en un servicio médico-quirúrgico de un hospital universitario}

\author{
BRAULIO ALIAGA ${ }^{a}$, NATALIA MOLINA ${ }^{a}$, MATÍAS NOGUERA ${ }^{a}$, \\ PAULA ESPINOZA ${ }^{a}$, SEBASTIÁN SÁNCHEZ ${ }^{a}$, BÁRBARA LARA ${ }^{1}$, \\ MARCELA CARRASCO ${ }^{1,2}$, GONZALO EYMIN $^{1,3}$
}

\section{Risk of falls among patients admitted to a medical-surgical ward. Analysis of 376 medical records}

\begin{abstract}
Background: Detecting patients at risk of falls during hospital stay is of utmost importance to implement preventive measures. Aim: To determine the frequency of patients with a high risk of falls admitted to a medical-surgical ward. To assess the preventive measures implemented. Materials and Methods: Review of medical records of 376 patients aged 20 to 97 years (28\% older than 70 years) admitted to a clinical hospital in a period of four months. Results: Eleven percent of patients had a history of falls, 50\% had a sensory deficit, $68 \%$ had unstable gait, $8 \%$ had a neurological risk condition, $8 \%$ had drowsiness or disorientation, $4 \%$ had psychomotor agitation or delirium, $86 \%$ used high risk medications, $73 \%$ used 2 or more high risk drugs and $72 \%$ were using devices that decrease mobility. One hundred forty-one patients (38\%) had a high risk of falling. The mean age of the latter was 77 years, $89 \%$ had a sensory deficit, 96\% had unstable gait, $4 \%$ had psychomotor agitation or delirium and 98\% used high risk drugs. Less than $1 \%$ had a medical prescription of a caregiver, physical restraints or antipsychotics, however, $21 \%$ of patients had a caregiver. Conclusions: The percentage of patients with a high risk of falling is important. The main risk factors were sensory deficit, unstable gait and the use of high risk medications. The low frequency of preventive measures prescriptions is striking.

(Rev Med Chile 2018; 146: 862-868)
\end{abstract}

Key words: Accidental Falls; Delirium, Risk Factors; Inpatients.

\section{L} as caídas, definidas como "una ida o descenso involuntaria hacia el suelo u otro nivel inferior", son un problema serio en los hospitales ${ }^{1}$. Se asocian a complicaciones graves que incluyen fracturas y pérdida de movilidad, prolongación de la estadía hospitalaria y problemas médico legales. Se estima que un tercio de las caídas son prevenibles con programas de evaluación de riesgo. Sin embargo, aún es un desafío el predecir las caídas intrahospitalarias ${ }^{2,3}$. En Chile, entre $2 \%$ y $12 \%$ de los pacientes hospitalizados sufren caídas, sin embargo, no existen datos de prevalencia de pacientes de alto riesgo de caídas (PARC) en servicios médico-quirúrgicos de baja complejidad (MQ) ${ }^{4}$. Un hospital de Estados Unidos de Norteamérica reportó 1,7 caídas anuales por cama en promedio, cifra mucho mayor a las caídas en la comunidad, donde se reportó un 
promedio de 0,65 caídas por cama anuales ${ }^{5}$. De acuerdo al tercer reporte del Patient Safety Observatory de caídas en hospitales, los costos asociados a las caídas ascienden a 20,7 millones de dólares americanos cada año (costo anual de 127,000 USD al año para un hospital de 800 camas $)^{6}$. Reconocer el riesgo de caídas en un paciente es fundamental para poder instaurar oportunamente medidas destinadas a prevenirlas, así como para disminuir el riesgo de daño secundario que pudiese alterar la rehabilitación y descompensar comorbilidades del paciente. Las caídas se relacionan, además, con ansiedad, depresión y síndrome postcaída, los cuales implican estadías hospitalarias más largas y una peor recuperación funcional ${ }^{7}$.

Existen distintas intervenciones para prevenir las caídas y lo principal para poder implementarlas es valorar el riesgo en cada paciente. Para esto existen escalas como la de Morse, de Downton, el modelo de riesgo de caída de Hendrich, la escala estratificada de Oliver, entre otras ${ }^{8-11}$. Sin embargo, ninguna de ellas ha mostrado identificar de manera óptima los PARC en el hospital.

En nuestra institución se utiliza una escala de valoración de riesgo de caídas adaptada localmente utilizando las escalas más usadas a nivel internacional (Tabla 1). Esta escala forma parte de la ficha clínica, se aplica a todos los pacientes del servicio MQ con una frecuencia diaria y evalúa los factores de riesgo conocidos de caídas intrahospitalarias: $\operatorname{Edad}^{12}$, antecedentes de caídas los últimos 3 meses $^{13}$, déficit sensorial ${ }^{11,15}$, condición neurológica de riesgo (psicosis, accidente vascular $[\mathrm{ACV}]$ convulsiones recientes, compromiso de conciencia) ${ }^{16}$, marcha inestable (marcha insegura o que requiere ayuda de terceros o de equipos de apoyo) ${ }^{17}$, estado cognitivo (somnolencia, desorientación, delirium o agitación psicomotora $)^{18}$, fármacos de alto riesgo de caídas (ARC) (benzodiacepinas, diuréticos, laxantes, antihipertensivos, anticoagulantes, hipnóticos, opiáceos, barbitúricos, antidepresivos, anticonvulsivantes, $\mathrm{y}$ antiparkinsonianos $)^{18}$, y uso de equipos que limiten la movilidad (sueros, sondas, catéter central de inserción periférica, etc. $)^{19}$. A partir de lo anterior, se generan categorías con distintos puntajes para catalogar a los pacientes como de alto riesgo de caída (al obtener un puntaje $\geq 5$ ) o de mediano o bajo riesgo. Cabe destacar que el delirium y la agitación psicomotora confieren por sí solos un alto riesgo de caídas (5 puntos). Todo el resto confiere 1 punto, salvo caídas previas en el hospital y la presencia de 2 o más fármacos de riesgo que confieren 2. De acuerdo al riesgo se implementan medidas de prevención y manejo: identificación mediante brazalete de PARC, uso de barandas, tratamiento de delirium, medidas de contención física, acompañamiento, o ambos, según corresponda.

Actualmente no existen estudios que evalúen la eficacia de los sistemas de contención en pacientes con patología aguda. El uso de sistemas de amarras que limitan la movilidad del paciente de alto riesgo se considera un método controvertido, de hecho, estudios demuestran que aumentan el daño asociado a las caídas ${ }^{19}$. La limitación del movimiento predispone a otros eventos adversos como delirium, úlceras por presión, incontinencia y daños severos como aspiración de vómito, estrangulación y muerte ${ }^{20}$. La presencia de cuidadores de pacientes con ARC tiene evidencia en disminuir las caídas ${ }^{21,22}$. La adherencia a las recomendaciones derivadas de la clasificación de riesgo de los pacientes no ha sido suficientemente analizada. El manejo de estos pacientes impone una fuerte carga a los cuidadores, al sistema de salud y a la familia y es por esto que es necesario revisar las estrategias de prevención de caídas a partir de una adecuada y oportuna detección de los factores de riesgo.

El objetivo de este trabajo es determinar el porcentaje de PARC en nuestro servicio MQ, estimar la frecuencia de indicación médica de medidas destinadas a reducir las caídas y su ejecución. De este modo podremos proyectar en el futuro estrategias de detección de PARC más sensibles y mejorar la indicación y ejecución de medidas preventivas.

\section{Materiales y Métodos}

Previa autorización por el Comité de Ética de la Facultad de Medicina de la Pontificia Universidad Católica de Chile, se revisaron de manera transversal a partir de registros clínicos (ficha médica, hoja de indicaciones y registros de enfermería) los factores de riesgo de caídas de la escala de valoración de riesgo institucional (Tabla 1), de todos los pacientes hospitalizados en un servicio Médico Quirúrgico del Hospital Clínico de la Red de Salud UC-Christus entre abril y julio de 2015, obteniéndose de cada paciente en una oportuni- 
Tabla 1. Escala institucional de riesgo de caídas

\begin{tabular}{|c|c|c|}
\hline Factores de riesgo (FR) & Variables (V) & Puntaje (P) \\
\hline \multirow[t]{2}{*}{ Edad } & $<75$ años & 0 \\
\hline & $>/=75$ años & 1 \\
\hline \multirow[t]{3}{*}{ Caídas previas } & Sin caídas & 0 \\
\hline & Casa & 1 \\
\hline & Hospitalizado & 2 \\
\hline \multirow[t]{2}{*}{ Déficit sensorial (visuales, auditivas y/o neuropáticas) } & Sin déficit sensorial & 0 \\
\hline & Con déficit sensorial & 1 \\
\hline \multirow{2}{*}{$\begin{array}{l}\text { Marcha inestable (marcha insegura y/o que requiere ayuda de terceros o } \\
\text { equipos de apoyo) }\end{array}$} & Sin marcha inestable & 0 \\
\hline & Con marcha inestable & 1 \\
\hline \multirow{2}{*}{$\begin{array}{l}\text { Condición neurológica/psiquiátrica de riesgo (compromiso de conciencia, } \\
\text { ACV reciente, crisis convulsiva reciente, psicosis, etc.) }\end{array}$} & Sin condición de riesgo & 0 \\
\hline & Con condición de riesgo & 1 \\
\hline \multirow[t]{2}{*}{ Estado cognitivo } & Somnoliento y/o desorientado & 1 \\
\hline & $\begin{array}{l}\text { Agitación psicomotora o } \\
\text { diagnóstico médico de delirium }\end{array}$ & 5 \\
\hline $\begin{array}{l}\text { No de medicamentos de riesgo (diurético, laxante, anticoagulante, } \\
\text { hipotensores, opiáceos, hipnóticos, barbitúricos, antidepresivos, } \\
\text { benzodiacepinas, antiparkinsonianos y antiepilépticos) }\end{array}$ & $\begin{array}{l}1 \text { medicamento } \\
2 \text { o más medicamentos }\end{array}$ & 1 \\
\hline \multirow{2}{*}{$\begin{array}{l}\text { Uso de equipos que limiten la movilidad al paciente (bombas de infusión, } \\
\text { catéteres, sondas, etc.) }\end{array}$} & No usa equipos & 0 \\
\hline & Si usa equipos & 1 \\
\hline
\end{tabular}

${ }^{*} \mathrm{ACV}$ : Accidente cerebrovascular.

dad los siguientes datos: Edad, antecedentes de caídas los últimos 3 meses, déficit sensorial (visual, auditivo), condición neurológica de riesgo (compromiso de conciencia, ACV reciente, crisis convulsiva reciente, psicosis), marcha inestable (marcha insegura o que requiere ayuda de terceros o equipos de apoyo), estado cognitivo (somnoliento o desorientado, agitación psicomotora o diagnóstico médico de delirium), fármacos de ARC (diuréticos, laxantes, anticoagulantes, hipotensores, opiáceos, hipnóticos, barbitúricos, antidepresivos, benzodiacepinas, antiparkinsonianos y antiepilépticos), y uso de equipos que limiten la movilidad (sonda urinaria, infusiones intravenosas continuas, líneas centrales de inserción periférica, sistemas de cierre de heridas por presión negativa). En pacientes clasificados como de ARC, se indagó sobre la indicación médica de contención física, de antipsicóticos, de cuidadora y la presencia o no de esta última. Se realizó un análisis descriptivo de todas las variables recolectadas y se reportaron como promedio \pm desviación estándar, mediana y porcentajes según la distribución de las variables. Las comparaciones de subgrupos se hicieron usando t-test, $\chi^{2}$, prueba exacta de Fisher y test de Mann-Whitney, según fuera apropiado. Todos los análisis fueron realizados en STATA versión 13.3.

\section{Resultados}

En 376 pacientes se encontraron 141 PARC $(37,5 \%)$. La edad promedio fue de 60,4 años (DS \pm 20 años, 18 a 97 años), $28 \%$ mayores de 70 años. Once por ciento tenía antecedentes de caídas, 50,3\% de déficit sensorial, $68 \%$ de marcha inestable, $8,5 \%$ de condición neurológica de riesgo, $7,8 \%$ de somnolencia o desorientación, $4,2 \%$ de agitación psicomotora o delirium. El 85,8\% usaba fármacos de riesgo (73,1\% de ellos usaba 2 o más), y 71,6\% usaba equipos que disminuyen la movilidad (Tabla 2). En menos de $1 \%$ de los 
Riesgo de caídas en unidades médico quirúrgicas - B. Aliaga et al

Tabla 2. Prevalencia de factores de riesgo de caídas en el total de pacientes

\begin{tabular}{|lc|}
\hline Factor de riesgo de caídas & Prevalencia \\
\hline Edad > 70 años & $28,00 \%$ \\
\hline Caídas previas & $11,00 \%$ \\
\hline Déficit sensorial & $50,30 \%$ \\
\hline Marcha inestable & $68,00 \%$ \\
\hline Condición neurológica o psiquiátrica de riesgo & $8,50 \%$ \\
\hline Agitación psicomotora o delirium & $4,20 \%$ \\
\hline 1 o más medicamentos de riesgo & $85,80 \%$ \\
\hline Uso de equipos que limitan movilidad & $71,60 \%$ \\
\hline
\end{tabular}

PARC se cumplía con el registro de la indicación médica de cuidadora. En el subgrupo de PARC, la edad promedio fue de $76,6 \pm 14$ años, $88,6 \%$ tenía déficit sensorial, 96,2\% marcha inestable, $3,7 \%$ agitación psicomotora o delirium y $98,1 \%$ usaba fármacos de riesgo. Los PARC tuvieron significativamente más antecedentes de caídas, uso de fármacos de alto riesgo, déficit sensorial, marcha inestable, condición neurológica de riesgo, somnolencia o desorientación que los pacientes de mediano y bajo riesgo de caídas $(\mathrm{p}<0,05)($ En la Tabla 3 y Figura 1 se comparan la presencia de factores de riesgo).

Tabla 3. Comparación factores de riesgo en pacientes con y sin alto riesgo de caídas

\begin{tabular}{|lccc|}
\hline Factor de Riesgo (FR) & $\begin{array}{c}\text { Con alto riesgo de } \\
\text { caídas (ARC) }\end{array}$ & $\begin{array}{c}\text { Sin alto riesgo de } \\
\text { caídas (SARC) }\end{array}$ & Valor p \\
\hline Caídas previas & $20,7 \%$ & $5,7 \%$ & 0,006 \\
\hline Déficit sensorial & $88,7 \%$ & $27,3 \%$ & 0,000 \\
\hline Marcha inestable & $96,2 \%$ & $51,7 \%$ & 0,000 \\
\hline Condición neurológica o psiquiátrica de riesgo & $17 \%$ & $3,4 \%$ & 0,005 \\
\hline Somnoliento y/o desorientado & $13,2 \%$ & $1,1 \%$ & 0,003 \\
\hline Agitación psicomotora o delirium & $3,8 \%$ & $0 \%$ & 0,066 \\
\hline 1 o más medicamentos de riesgo & $98,1 \%$ & $78,4 \%$ & 0,001 \\
\hline Uso de equipos que limitan movilidad & $68 \%$ & $75 \%$ & 0,463 \\
\hline
\end{tabular}

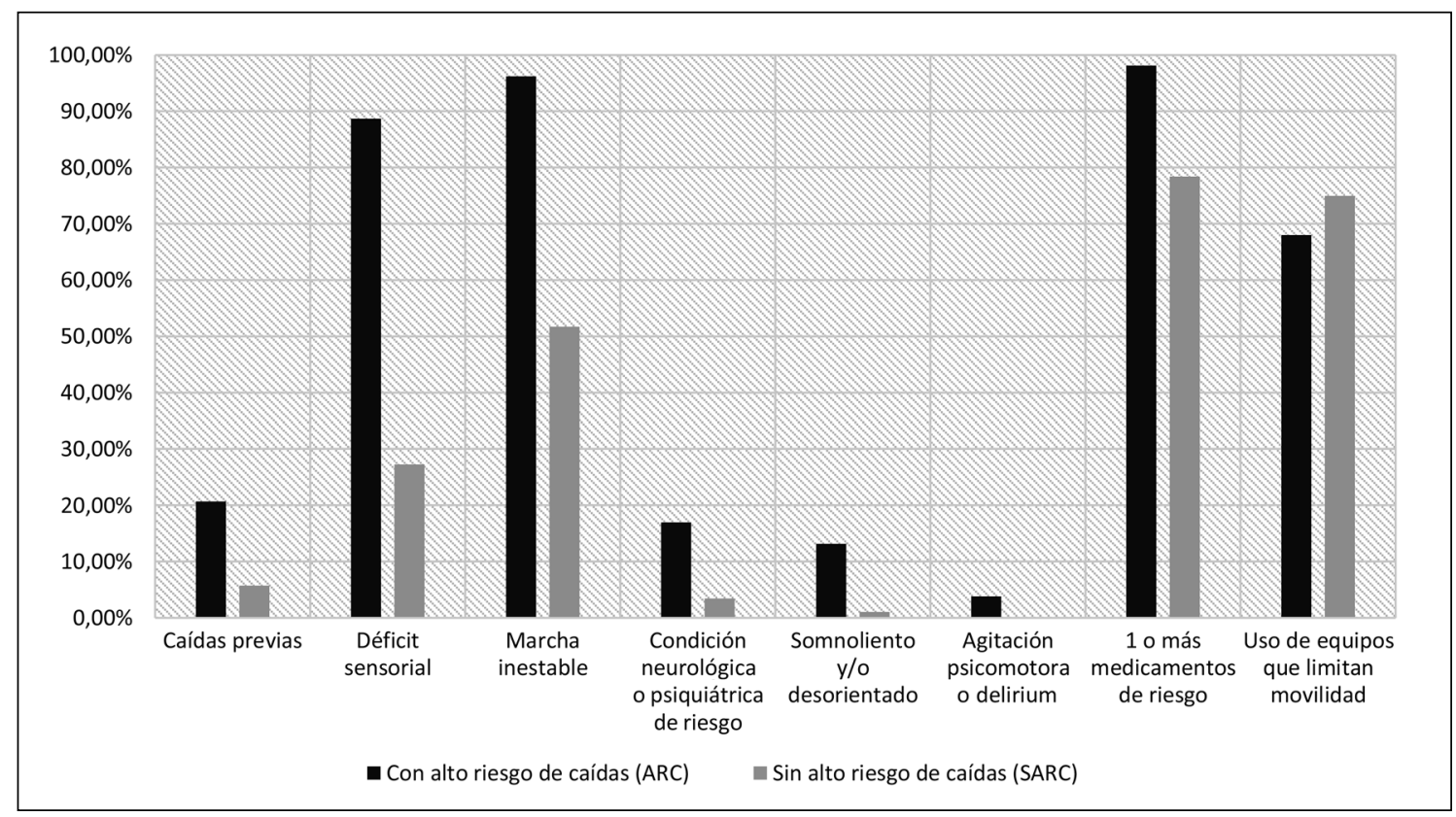

Figura 1. Comparación factores de riesgo en pacientes con y sin alto riesgo de caídas. 


\section{Discusión}

La tasa de caídas de 2015 en nuestro servicio MQ fue de 1/1.000 días cama ocupados ( 8 en 8.073 días cama). Puede que esta cifra esté subestimada por un bajo nivel de reporte por parte de las enfermeras, ya sea por temor, por falta de tiempo o por considerar que la caída fue menor y sin daño. La prevalencia de PARC en nuestro servicio médico-quirúrgico fue más alta que la reportada por otros estudios, como por ejemplo el estudio de Kobayashi et al, donde se encontró 10,3\% de PARC en una serie de 163.558 pacientes, que incluía pacientes hospitalizados en unidades de mediana y alta complejidad, y si bien el instrumento de categorización de riesgo es diferente al nuestro, los parámetros utilizados son muy parecidos ${ }^{20}$. $\mathrm{Al}$ ser nuestra escala de valoración de riesgo una adaptación local de los diferentes instrumentos validados en la literatura, hay que ser cauto a la hora de comparar con otros estudios. No es raro que la presencia de PARC sea alta en nuestro servicio considerando el envejecimiento de la población y la mayor prestación de servicios ambulatorios en pacientes jóvenes y con menos comorbilidad. De acuerdo a lo encontrado en estudios de PARC, el factor de riesgo que más se relaciona con caídas en el hospital, es el antecedente de caídas previas ${ }^{23}$. Sin embargo, en nuestra serie, el antecedente de caídas previas se encontró en solo $20 \%$ de los PARC, proporción bastante menor al déficit sensorial $(88,7 \%)$, a la marcha inestable $(96,2 \%)$ y al uso de fármacos de riesgo $(98,1 \%)$. Otro importante factor de riesgo analizado es la presencia de delirium o agitación psicomotora, siendo nuestra incidencia $(4,2 \%)$ similar a la reportada en el trabajo de Van Velthuijsen, quien encontró 5\% de pacientes con delirium en su serie $(23 \%$ de estos fue delirium hipoactivo ${ }^{24}$. Probablemente la incidencia de delirium encontrada en nuestra cohorte esté subvalorada debido a que al momento del estudio no se utilizaba una escala de tamizaje de delirium en forma protocolizada y los niveles de subdiagnóstico son muy significativos cuando no se hace una búsqueda dirigida de este. Se estiman incidencias de delirium entre 11 y $14 \%$ en pacientes en este tipo de unidades ${ }^{25}$. Por otra parte, se encontró que en menos de $1 \%$ de los pacientes estaba escrita la indicación de cuidadora o medidas de contención farmacológica o física en PARC por parte del médico. Esta baja adherencia al registro de las medidas de prevención de caídas podría deberse a una baja percepción del riesgo por parte del equipo médico o a la poca disponibilidad de cuidadoras, ya que la mayor parte de las veces las medidas de prevención de caídas son tomadas por las enfermeras y son aplicadas aunque no sean registradas por el médico, como sucede cuando la enfermera le pide a la familia que cumpla labor de acompañante aunque esto no esté registrado. Sin embargo, en otras medidas, como las contenciones, dado que implican un riesgo potencial, es fundamental asegurar la indicación médica.

Nuestros resultados resaltan la importancia de estar alerta al potencial alto riesgo de caídas en pacientes con uso de fármacos de alto riesgo, con déficit sensorial, marcha inestable, somnolientos, desorientados o portadores de una condición neurológica de riesgo. No pretendemos mostrar la incidencia de caídas en la cohorte ni su asociación con los factores de riesgo, puesto que esto ya está validado en otros estudios ${ }^{8-11}$. Nuestra escala de riesgo institucional deriva de una adaptación local de varios instrumentos, y se basa en factores de riesgo plenamente validados en la literatura. Este trabajo es innovador en cuantificar el porcentaje de PARC en un servicio MQ y los factores de riesgo más frecuentes en ellos. El alto porcentaje de PARC nos invita a reflexionar si estos pacientes deberían admitirse a unidades de cuidados intermedios por dependencia más que por complejidad médica, o bien si los servicios médico quirúrgicos deberían rediseñarse y contar con más personal de apoyo acorde a las necesidades de mayor cuidado y dependencia de este grupo de pacientes. Esta última estrategia suena más razonable que mantener a los PARC en unidades de mayor complejidad solo por vigilancia, medida que a la vez de ser de alto costo se contrapone con la escasez de camas críticas ${ }^{26}$. La herramienta CUDYR, creada por facultativos de esta universidad, incorpora la variable "necesidad de vigilancia del paciente" al momento de definir la unidad de destino del paciente ${ }^{27}$. Esta herramienta categoriza a los usuarios de modo de conocer el perfil ocupacional de cada servicio, posibilitando la identificación de las cargas de trabajo del personal de enfermería y, de este modo, asignar los recursos sobre una base de elementos objetivos. La utilización de este método ha hecho factible la comparación de las dotaciones de personal de los servicios, con los 
requerimientos de los pacientes según su nivel de riesgo y dependencia, manteniendo constante la calidad de la asistencia y garantizando la seguridad de la atención. Si bien la incidencia de caídas en este servicio en particular es baja, las implicancias que pueden tener las caídas obligan a extremar las medidas para que estas no sucedan ${ }^{3,5}$. La aplicación precoz de esta herramienta bajo las condiciones actuales de nuestros servicios MQ podría ser útil para definir a qué unidad debe ingresar un determinado paciente, con el fin de reducir efectivamente las posibilidades de sufrir una caída. Esta herramienta podría ser aplicada por enfermeras de urgencias, admisión y unidades de paciente crítico al momento de definir a qué área debe ir un paciente en particular (puesto que es este estamento el que define la cama de destino del paciente). Con la información existente no sabemos si es costo efectivo aumentar las dotaciones de personal paramédico en los servicios de baja complejidad con el fin de evitar las caídas.

La medida que ha demostrado mayor efectividad en reducir el riesgo de caídas intrahospitalarias es la implementación de protocolos de prevención de delirium. Estos protocolos se basan en el manejo de los precipitantes más frecuentes de delirium e incluyen: evitar la deshidratación, favorecer la movilidad, el descanso y el sueño nocturno, evitar medicamentos de alto riesgo de delirium (sedantes, antipsicóticos, anticolinérgicos, entre otros), favorecer la estimulación sensorial y cognitiva orientando con adecuada luz en el día y oscuridad en la noche, estimular a que usen sus lentes y audífonos, si los requieren, y favorecer el acompañamiento, idealmente por un familiar que contribuya a que los puntos anteriores se logren. Estas medidas logran reducir en 50\% las chances de presentar delirium y $62 \%$ de las caídas intrahospitalarias ${ }^{28}$.

\section{Conclusión}

Más de un tercio de los pacientes admitidos a nuestro servicio MQ tiene un alto riesgo de caídas, de ahí que los servicios deben adaptarse a un perfil nuevo de usuario, con un mayor nivel de dependencia y requirente de mayor asistencia para disminuir eventos adversos como son las caídas. Es importante implementar herramientas sensibles y sencillas destinadas a una detección precoz de PARC de manera periódica, de modo de poder implementar medidas efectivas de manera oportuna. En este sentido es importante aplicar herramientas de prevención y de detección del delirium de manera sistemática. Mientras no se mejoren las condiciones de los servicios MQ para acoger de manera segura a estos pacientes, las caídas y sus consecuencias podrían evitarse con una aproximación interdisciplinaria en la que participen médicos, enfermeras, personal paramédico, kinesiólogos, cuidadores y familiares. Esto implica un cambio cultural en la manera como se manejan los pacientes hoy en los hospitales, pero dado que la evidencia es contundente, es crucial avanzar en mejorar la calidad asistencial y evitar los riesgos asociados a la hospitalización, entre los que se encuentran las caídas y sus graves consecuencias.

\section{Referencias}

1. Buchner DM, Hornbrook MC, Kutner NG, Tinetti ME, Ory MG, Mulrow CD, et al. Development of the common data base for the FICSIT trials. J Am Geriatr Soc 1993;41: 297-308.

2. Jencks SF, Williams MV, Coleman EA. Rehospitalizations among patients in the Medicare fee-for-service program. N Engl J Med 2009; 360 (14): 1418-28.

3. Joint Commission International Accreditation Standards for Hospitals. 3rd ed. Standards only. 32. 2007. Accedido el 3 de abril de 2018. Disponible en: https:// www.jointcommissioninternational.org/assets/3/7/ Hospital-5E-Standards-Only-Mar2014.pdf.

4. Ministerio de Salud. Normas sobre la seguridad de la atención del paciente y calidad de la atención respecto de: Reporte de caídas de pacientes hospitalizados. Accedido el 7 de marzo de 2018. Disponible en: http://www. supersalud.gob.cl/observatorio/671/articles-8928_recurso_8.pdf.

5. Bergen $G$, Stevens MR, Burns ER. Falls and Fall Injuries Among Adults Aged $\geq 65$ Years-United States, 2014 . Morb Mortal Wkly Rep 2016; 65: 993-8.

6. Slips, trips and falls in hospitals. The Third Report from the Patient Safety Observatory. National Patient Safety Agency. Accedido el 7 de marzo del 2018. Disponible en: www.npsa.nhs.uk/EasySiteWeb/GatewayLink.aspx? alId $=6242$.

7. Vetter N, Ford D. Anxiety and depression scores in elderly fallers. Int J Geriatr Med 1989; 4: 159-63.

8. Morse J, Black C, Oberle K, Donahue P. A prospective 
study to identify the fall-prone patient. Soc Sci Med 1989; 28 (1): 81-6.

9. Bueno-García M, Roldán-Chicano M, Rodríguez-Tello J, Meroño-Rivera M, Dávila-Martínez R, Berenguer-García N. Characteristics of the Downton fall risk assessment scale in hospitalised patients. Enferm Clin 2017; 27 (4): 227-34.

10. Hendrich AL, Bender PS, Nyhuis A. Validation of the Hendrich II fall risk model: a large concurrent case/ control study of hospitalized patients. Appl Nurs Res 2003; 16: 9-21.

11. Oliver D, Britton M, Seed P, Martin FC, Hopper A. Development and evaluation of evidence based risk assessment tool (STRATIFY) to predict which elderly inpatients will fall: case-control and cohort studies. BMJ 1997; 315: 1049-53.

12. Guo Z, Wills P, Viitanen M, Fastbom J, Winblad B. Cognitive impairment, drug use, and the risk of hip fracture in persons over 75 years old: a community-based prospective study. Am J Epidemiol 1998; 148: 887.

13. Nevitt MC, Cummings SR, Kidd S, Black D. Risk factors for recurrent nonsyncopal falls. A prospective study. JAMA 1989; 261: 2663.

14. Viljanen A, Kaprio J, Pyykkö I, Sorri M, Pajala S, Kauppinen M, et al. Hearing as a predictor of falls and postural balance in older female twins. J Gerontol A Biol Sci Med Sci 2009; 64: 312.

15. Felson DT, Anderson JJ, Hannan MT, Milton RC, Wilson PW, Kiel DP. Impaired vision and hip fracture. The Framingham Study. J Am Geriatr Soc 1989; 37: 495.

16. Ferrucci L, Bandinelli S, Cavazzini C, Lauretani F, Corsi A, Bartali B, et al. Neurological examination findings to predict limitations in mobility and falls in older persons without a history of neurological disease. Am J Med 2004; 116: 807.

17. De Rekeneire N, Visser M, Peila R, Nevitt MC, Cauley JA, Tylavsky FA, et al. Is a fall just a fall: correlates of falling in healthy older persons. The Health, Aging and Body Composition Study. J Am Geriatr Soc 2003; 51: 841.

18. Mion LC, Chandler AM, Waters TM, Dietrich MS, Kess- ler LA, Miller ST, et al. Is it possible to identify risks for injurious falls in hospitalized patients? Jt Comm J Qual Patient Saf 2012; 38 (9): 408-13.

19. Evans D, Wood J, Lambert L. Patient injury and physical restraint devices: a systematic review. J Adv Nurs 2003; 41 (3): 274-82.

20. Kobayashi K, Imagama S, Ando K, Inagaki Y, Suzuki $\mathrm{Y}$, Nishida $\mathrm{Y}$, et al. Analysis of falls that caused serious events in hospitalized patients. Geriatr Gerontol Int 2017; 17 (12): 2403-240.

21. Donoghue J, Graham J, Mitten-Lewis S, Murphy M, Gibbs J. A volunteer companion-observer intervention reduces falls on an acute aged care ward. Int J Health Care Qual Assur Inc Leadersh Health Serv 2005; 18 (1): 24-31.

22. Giles LC, Bolch D, Rouvray R, McErlean B, Whitehead $\mathrm{CH}$, Phillips PA, Crotty M. Can volunteer companions prevent falls among inpatients? A feasibility study using a pre-post comparative design. BMC Geriatr 2006; 6: 11.

23. Deandrea S, Bravi F, Turati F, Lucenteforte E, La Vecchia C, Negri E. Risk factors for falls in older people in nursing homes and hospitals. A systematic review and meta-analysis. Arch Gerontol Geriatr 2013; 56 (3): 40715.

24. Van Velthuijsen E, Zwakhalen S, Mulder W, Verhey F, Kempen G. Detection and management of hyperactive and hypoactive delirium in older patients during hospitalization: a retrospective cohort study evaluating daily practice. Int J Geriatr Psychiatry. 2017.

25. Inouye $S$, Westendorp R, Saczynski J. Delirium in elderly people. Lancet 2014; 383 (9920): 911-22.

26. Fonasa. Acuerdos GRD (Grupo relacionados a diagnóstico). Accedido el 4 de agosto de 2017. Disponible en: https://www.fonasa.cl/sites/fonasa/prestadores/convenios/red-publica/prestadores-publicos/acuerdos-grd.

27. García M, Castillo L. Categorización de usuarios: Una herramienta para evaluar las cargas de trabajo de enfermería. Rev Med Chile 2000; 128 (2): 177-83.

28. Hshieh TT, Yue J, Oh E, Puelle M, Dowal S, Travison T, et al. Effectiveness of Multicomponent Nonpharmacological Delirium Interventions A Meta-analysis. JAMA Intern Med 2015; 175 (4): 512-20. 